\title{
The equivalence principle in Kaluza-Klein gravity
}

\author{
J. Ponce de Leon* \\ Laboratory of Theoretical Physics, Department of Physics \\ University of Puerto Rico, P.O. Box 23343, San Juan, \\ PR 00931, USA
}

April 2008

\begin{abstract}
In four-dimensional general relativity the spacetime outside of an isolated spherical star is described by a unique line element, which is the Schwarzschild metric. As a consequence, the "gravitational" mass and the "inertial" mass of a star are equal to each other. However, theories that envision our world as embedded in a larger universe, with more than four dimensions, permit a number of possible non-Schwarzschild $4 D$ exteriors, which typically lead to different masses, violating the weak equivalence principle of ordinary general relativity. Therefore, the question arises of whether the violation of this principle, i.e., the equality of gravitational and inertial mass, is a necessary consequence of the existence of extra dimensions. In this paper, in the context of Kaluza-Klein gravity in $5 D$, we show that the answer to this question is negative. We find a one-parameter family of asymptotically flat non-Schwarzschild static exteriors for which the inertial and gravitational masses are equal to each other, and equal to the Deser-Soldate mass. This family is consistent with the Newtonian weak-field limit as well as with the general-relativistic Schwarzschild limit. Thus, we conclude that the existence of an extra dimension, and the corresponding non-Schwarzschild exterior, does not necessarily require different masses. However, to an observer in $4 D$, it does affect the motion of test particles in $4 D$, which is a consequence of the departure from the usual $(4 D)$ law of geodesic motion.
\end{abstract}

PACS: 04.50.+h; 04.20.Cv

Keywords: Kaluza-Klein Theory; General Relativity; Space-Time-Matter theory.

*E-mail: jpdel@ltp.upr.clu.edu; jpdel1@hotmail.com 


\section{Introduction}

According to Birkhoff's theorem, in general relativity the metric of the spacetime in the region surrounding an isolated spherical star is given by the Schwarzschild vacuum solution. The uniqueness of this solution has played an important role in our understanding of relativistic stars and gravitational collapse. Perhaps, the most significant feature of stars in general relativity is the equality between the "gravitational" mass $M_{g}$ and the "inertial" mass $M_{\text {in }}$.

The "gravitational" mass is the one that determines the gravitational field produced by the body; this is the mass that appears in the metric tensor in a gravitational field, or, in particular, in Newton's gravitational law. Although, the concept of gravitational mass in a given region of space is not available in general situations, for a constant gravitational field ( $\equiv$ time-translational symmetry) it is possible to derive a simple expression for the total energy of matter plus field in the form of an integral over the space occupied by the matter. This is the well-known Tolman-Whittaker formula [1]

$$
M_{g}=\int\left(T_{0}^{0}-T_{1}^{1}-T_{2}^{2}-T_{3}^{3}\right) \sqrt{-g_{4}} d V_{3} .
$$

In the case of central symmetry of the field there is another expression for this quantity, namely

$$
m=\int T_{0}^{0} d V_{3} .
$$

Since this is the mass that acts on tests particles it can also be obtained from the analysis of the geodesic equation. The "inertial" mass is the one entering in Newton's second law, it is identified with the zeroth component of the four-momentum vector $P^{\mu}$ of the body, viz.,

$$
M_{\text {in }}=P^{0},
$$

and is calculated from the asymptotic behavior of the spatial part of the metric.

In fact, the equality

$$
M_{g}=M_{i n}=m,
$$

constitutes a fundamental principle in general relativity and is known as the (weak) equivalence principle. In practice, it means that objects of different nature fall at the same rate in a gravitational field.

Nowadays, there are various theories that invoke the existence of extra dimensions, although with different purposes and motivations. The study of the stellar structure, in the context of these theories, might constitute an important approach for predicting measurable effects from the putative extra dimensions. Thus far, it has been found that the effective four-dimensional picture allows the existence of different possible, non-Schwarzschild, scenarios for the description of the spacetime outside of a spherical star.

In braneworld models, Germani and Maartens 2] have found two exact vacuum solutions, both asymptotically Schwarzschild, which can be used to represent the exterior of an uniform-density star. Bruni, Germani and Maartens [3] have shown that the vacuum exterior of a collapsing dust cloud cannot be static. Similar results have been derived by Kofinas and Papantonopoulos 44 in the context of various braneworld models with curvature corrections. Other possible non-Schwarzschild stellar exteriors, with spatial spherical symmetry, have been discussed by Dadhich et al [5], Casadio et al [6], Viser and Wiltshire [7, and Bronnikov et al [8. As a consequence the stellar structure in braneworld theory is rather different from that in ordinary general relativity. In particular, the gravitational and inertial masses are not necessarily equal. As an illustration, let us consider a spherical braneworld star whose region outside the surface is described by the "spatial Schwarzschild" metric

$$
d s^{2}=\frac{1}{b^{2}}\left(b-1+\sqrt{1-\frac{2 b \mathcal{M}}{R}}\right)^{2} d t^{2}-\left(1-\frac{2 b \mathcal{M}}{R}\right)^{-1} d R^{2}-R^{2} d \Omega^{2},
$$

which for $b=1$ reduces to the Schwarzschild vacuum metric. The total gravitational mass of such a star is $\mathcal{M}$, i.e., $M_{g}=\mathcal{M}$. On the other hand, the total inertial mass is $M_{i n}=b \mathcal{M}$; only in the Schwarzschild case $M_{g}=M_{i n}=\mathcal{M}$.

An analogous situation occurs in Kaluza-Klein gravity. Specifically, there are a number of asymptotically flat, non-Schwarzschild exteriors that can continuously be matched with the interior of a spherical star [9]. Regarding 
the equivalence principle, in Kaluza-Klein theory one should also consider an alternative definition of total mass, proposed by Deser and Soldate [10, which explicitly contains the contribution from the extra dimensions.

The object of this work is to show that the existence of an extra dimension, and non-Schwarzschild exterior, does not necessarily imply a violation of the weak equivalence principle. In order to avoid misunderstanding, we should emphasize that in the present work we examine this principle in the sense where it refers to the equality of definitions for mass (4). With this aim we test this equality within the framework of a family of static spherically symmetric metrics, which are possible static stellar exteriors [9], in five-dimensional Kaluza-Klein gravity. Then, we discuss and calculate the Deser-Soldate mass, say $M_{D S}$, for these models. Within this framework, we find a one-parameter family of asymptotically flat non-Schwarzschild exteriors for which (as in ordinary general relativity) the masses are all equal, i.e.,

$$
M_{g}=M_{i n}=m=M_{D S} .
$$

in agreement with the (weak) equivalence principle.

\section{Description of the model}

In Kaluza-Klein gravity, there is a family of Ricci-flat solutions $\left(R_{A B}=0\right)$, which can be considered as the natural generalization of the Schwarzschild spacetime (see a recent discussion by Lake [11, and references therein). In five-dimensions, in the form given by Davidson and Owen [12], these solutions are described by the line element 1 ]

$$
d S^{2}=\left(\frac{\bar{a} \rho-1}{\bar{a} \rho+1}\right)^{2 \sigma k} d t^{2}-\frac{1}{\bar{a}^{4} \rho^{4}} \frac{(\bar{a} \rho+1)^{2[\sigma(k-1)+1]}}{(\bar{a} \rho-1)^{2[\sigma(k-1)-1]}}\left[d \rho^{2}+\rho^{2} d \Omega^{2}\right] \pm\left(\frac{\bar{a} \rho+1}{\bar{a} \rho-1}\right)^{2 \sigma} d y^{2},
$$

where $\bar{a}$ is a constant with dimensions of $L^{-1}$; and $\sigma$ as well as $k$ are parameters that obey the constraint

$$
\sigma^{2}\left(k^{2}-k+1\right)=1 .
$$

In the original Kaluza-Klein theory, where the extra-dimensions are assumed to be compact and small, the higher dimensional gravity is reduced down to four dimensions by integrating out heavier $K K$ gravitational models, see for example Ref. [15. However, today it is amply accepted that the putative extra dimension can be large, in principle $-\infty<y<\infty$. In this new framework, the popular wisdom is that we recover our $4 D$ world by going onto a subspace $y=$ constant where the induced metric, say $g_{\mu \nu}^{(i n d)}$, coincides with the spacetime part of (7). Namely,

$$
d s_{i n d}^{2}=\left(\frac{\bar{a} \rho-1}{\bar{a} \rho+1}\right)^{2 \sigma k} d t^{2}-\frac{1}{\bar{a}^{4} \rho^{4}} \frac{(\bar{a} \rho+1)^{2[\sigma(k-1)+1]}}{(\bar{a} \rho-1)^{2[\sigma(k-1)-1]}}\left[d \rho^{2}+\rho^{2} d \Omega^{2}\right] .
$$

In the limit $k \rightarrow \infty(\sigma k \rightarrow 1)$, it reproduces the Schwarzschild metric, in isotropic coordinates, for a central mass $M=2 / \bar{a}$, viz.,

$$
d s_{S c h w}^{2}=d s_{i n d \mid k \rightarrow \infty}^{2}=\left(\frac{1-M / 2 \rho}{1+M / 2 \rho}\right)^{2} d t^{2}-\left(1+\frac{M}{2 \rho}\right)^{4}\left[d \rho^{2}+\rho^{2} d \Omega^{2}\right] .
$$

In the context of Space-Time-Matter theory (STM), metric (9) is interpreted as describing extended spherical objects called solitons [16]-21] (for a recent discussion see Ref. [22] and references therein). However, in Kaluza-Klein gravity there is another possible interpretation, which is suggested by the fact that (9) is singular at $\rho=1 / \bar{a}$, and asymptotically flat for $\bar{a} \rho \gg 1$. Namely, that (9) can be used to describe the effective $4 D$ spacetime outside of a spherical star embedded in $5 D$. In this interpretation, the effective exterior is not empty because there are nonlocal stresses induced from the Weyl curvature in $5 D$, which in $4 D$ behave like radiation 3 .

\footnotetext{
${ }^{1}$ This family of solutions has been rediscovered in different forms by Kramer [13] and, although in a different context, by Gross and Perry [14.

${ }^{2}$ In general, this is a naked lightlike singularity. Only in the Schwarzschild limit $k \rightarrow \infty(\sigma k \rightarrow 1)$ the $4 D$ metric $(9)$ possesses an event horizon.

${ }^{3}$ It should be noted that all non-Schwarzschild exteriors discussed in the literature 2 8 - 8 he a non-vanishing effective energymomentum tensor, which is defined through the conventional Einstein equations
} 
Following this line of reasoning, the "simplest" approach is to identify the induced metric (9) with the effective $4 D$ metric outside of a spherical star, which we denote as $g_{\mu \nu}^{(e f f)}$. However, in 9 ] we showed that this simple approach leads to contradictions in the Newtonian limit. Therefore, we extended the discussion by considering, as a possible exterior for a static spherical star, a family of asymptotically flat metrics which are conformal to (9). In particular, we showed that

$$
d s^{2}=\left(\frac{\bar{a} \rho-1}{\bar{a} \rho+1}\right)^{2 \varepsilon} d t^{2}-\frac{1}{\bar{a}^{4} \rho^{4}} \frac{(\bar{a} \rho+1)^{2[\varepsilon+1]}}{(\bar{a} \rho-1)^{2[\varepsilon-1]}}\left[d \rho^{2}+\rho^{2} d \Omega^{2}\right],
$$

is the only line element, that is consistent with both: the Newtonian limit for any value of $\varepsilon$, and the Schwarzschild limit, which is obtained for $\varepsilon=1$. In section 3 we will show that this line element is also consistent with the equivalence principle.

\subsection{The $5 D$ solution in Schwarzschild-like coordinates}

In order to make contact with other works in the literature, it is convenient to introduce a system of coordinates where the $5 D$ solutions (7) resemble the $4 D$ Schwarzschild solution in its usual form. With this aim we make the coordinate transformation

$$
r=\rho\left(1+\frac{1}{\bar{a} \rho}\right)^{2}
$$

which renders (7) into

$$
d S^{2}=A^{a} d t^{2}-A^{-(a+b)} d r^{2}-r^{2} A^{(1-a-b)}\left[d \theta^{2}+\sin ^{2} \theta d \phi^{2}\right] \pm A^{b} d y^{2},
$$

with

$$
A=\left(1-\frac{2 \mathcal{M}}{r}\right), \quad \mathcal{M}=\frac{2}{\bar{a}}, \quad a=\sigma k, \quad b=-\sigma .
$$

In this notation, the constraint (8) reads

$$
a^{2}+a b+b^{2}=1 .
$$

For the choice $a=1, b=0$ the $4 D$ part of (13) becomes identical to the Schwarzschild vacuum solution for a central mass $M=\mathcal{M}$.

\subsection{The effective exterior spacetime}

For the four-dimensional interpretation of (13), as in [9] we assume that the effective metric in $4 D$ is

$$
g_{\mu \nu}^{(e f f)}=\Phi^{N} g_{\mu \nu}^{(i n d)},
$$

where $N$ is an arbitrary real constant; $g_{\mu \nu}^{(i n d)}$ is the metric induced on the subspace $y=$ constant, and $\Phi=A^{b / 2}$. This ansatz is not new; it consolidates various approaches in the literature. In the context of Kaluza-Klein gravity and STM, it has been considered by Wesson [17, Kokarev [23-[24], Sajko 25], and the present author [26]. For $N=0$, it reproduces the usual interpretation of braneworld and STM theories. For $N=-2$ the interpretation is similar to the one provided by the canonical metric [27. For $N=1$ it yields the classical KK interpretation [15], which is used, in particular, by Davidson-Owen [12] and Dolan-Duff [28.

The resulting line element, for the effective exterior $4 D$ spacetime, can be written as

$$
d s_{(N)}^{2}=A^{\varepsilon} d t^{2}-A^{[-\varepsilon+b(N-1)]} d r^{2}-r^{2} A^{[(1-\varepsilon)+b(N-1)]}\left[d \theta^{2}+\sin ^{2} \theta d \phi^{2}\right],
$$

where

$$
\varepsilon=a+\frac{N b}{2},
$$


and consequently, from (15), we get

$$
b_{ \pm}=\frac{2 \varepsilon(N-1) \mp 2 \sqrt{3\left(1-\varepsilon^{2}\right)+(N-1)^{2}}}{\left(N^{2}-2 N+4\right)} .
$$

We take $b=b_{+}$for $N<1$, and $b=b_{-}$for $N>1$. In this way for $\varepsilon=1$ we recover the Schwarzschild solution. For $N=1$, the effective $4 D$ metric (17) does not depend on $b$.

For completeness, it is worth mentioning that, for a large extra dimension, both braneworld and STM theories are mathematically equivalent 29 . In particular, they both require slicing of the $5 D$ manifold in order to recover our $4 D$ spacetime. The fundamental difference between them is that in STM there is no matter inserted by hand into the $5 D$ manifold, while in the braneworld formalism it is postulated that there is an energy-momentum tensor in $5 D$ which is discontinuous at the thin-shell (the brane) that is assumed to represent our $4 D$ universe. In what follows we continue our discussion within the context of STM.

\subsection{The stellar interior}

Standard matching conditions allow to interpret the family of asymptotically flat metrics (17) as possible nonSchwarzschild scenarios for the spacetime outside of a spherical star for any $N$ and $\varepsilon$. In appendix A, we analyze the boundary conditions for the case where the stellar interior is described by a static spherical metric in curvature coordinates, viz.,

$$
d s^{2}=e^{\omega(R)} d t^{2}-e^{\sigma(R)} d R^{2}-R^{2}\left[d \theta^{2}+\sin \theta d \phi^{2}\right] .
$$

The boundary of the star is a "fixed" three-dimensional surface $\Sigma$ defined by the equation $R=R_{b}$ from inside, and $r=r_{b}$ from outsidet The constants $R_{b}$ and $r_{b}$ are related by

$$
R_{b}=r_{b}\left(1-\frac{2 \mathcal{M}}{r_{b}}\right)^{\gamma}
$$

where, in order to simplify the notation, we have set

$$
\gamma=1-\varepsilon+b(N-1)
$$

Boundary conditions ( $(\underline{\mathrm{A}-3}),(\underline{\mathrm{A}-6)}$ ) and $(\underline{\mathrm{A}-7})$, for the exterior metric under consideration, require

$$
e^{\omega\left(R_{b}\right)}=A^{\varepsilon}\left(r_{b}\right), \quad e^{-\sigma\left(R_{b}\right)}=A\left(r_{b}\right)\left(1+\frac{\gamma \mathcal{M}}{r_{b} A\left(r_{b}\right)}\right)^{2}, \quad \omega_{R}\left(R_{b}\right)=\frac{2 \varepsilon \mathcal{M}}{r_{b}^{2}} \frac{A\left(r_{b}\right)^{-(\gamma+2) / 2}}{\left[1+\gamma \mathcal{M} / r_{b} A\left(r_{b}\right)\right]},
$$

which impose no restrictions on parameters $(\varepsilon, N)$. We note that the embedding of the interior solution in $5 D$ neither restricts these parameters $[9$.

\subsection{Newtonian limit}

In the Newtonian limit we can write $e^{\omega(R)}=1+\xi f(R)$ and $e^{\sigma(R)}=1+\xi h(R)$, where $\xi$ is a "small" dimensionless parameter, i.e., $|\xi| \ll 1$. To first order in $\xi$, the gravitational mass $(\mathrm{A}-8)$ inside a sphere of radius $R$ is given by

$$
M_{g}(R)=\frac{\xi R^{2}}{2}\left(\frac{d f}{d R}\right) .
$$

In this approximation, $T_{0}^{0} \gg\left|T_{1}^{1}\right|, T_{0}^{0} \gg\left|T_{2}^{2}\right|=\left|T_{3}^{3}\right|$. Then, from (1) it follows that $M_{g}(R)=4 \pi \int_{0}^{R} \bar{R}^{2} T_{0}^{0}(\bar{R}) d \bar{R}$. Using the field equations we get

$$
8 \pi T_{0}^{0}=\frac{\xi}{R^{2}} \frac{d}{d R}(R h)+O\left(\xi^{2}\right) .
$$

\footnotetext{
${ }^{4}$ In the notation $R_{b}$ and $r_{b}$, "b" stands for boundary and has nothing to do with the parameter $b$ in metric (17).
} 
Consequently $M(R)=\xi R h / 2$. On the other hand we have (24). Thus, in this approximation $h=R(d f / d R)$. In terms of the original metric, the existence of a Newtonian limit requires.

$$
e^{\sigma(R)}-1=R \frac{d \omega}{d R}+O\left(\xi^{2}\right)
$$

inside the body, including its external boundary.

Let us now investigate in more detail the consequences of (26). For this we evaluate it at the boundary and use the matching conditions (23). For the case under consideration, the parameter $\xi$ can be taken as $\xi=\mathcal{M} / r$. Therefore, $A=1-2 \xi$ and $R=r\left[1-2 \gamma \xi+O\left(\xi^{2}\right)\right]$. Then, from (23) we find $e^{\sigma}=1-2 \xi(\gamma-1)+O\left(\xi^{2}\right)$ and $R \omega_{R}=2 \xi \varepsilon+O\left(\xi^{2}\right)$. Using (22), we find that the Newtonian limit (26) demands

$$
b(N-1)=0 .
$$

This condition is fulfilled in two cases: (i) $b=0$, for any arbitrary $N$ which corresponds to an exterior described by the Schwarzschild vacuum solution for $\varepsilon=1$; (ii) $N=1$ and $\varepsilon \neq 1$, in which case the exterior of a star is not an empty Schwarzschild spacetime.

\section{Calculating the different masses}

In this section we evaluate $M_{g}, M_{i n}$ and $m$ mentioned in the introduction by using the standard equations in $4 D$ for static gravitational fields given by (1) and (2). However, it is important to emphasize that using these equations we are not disregarding the contribution from the "extra" metric coefficient $g_{55}= \pm \Phi^{2}= \pm A^{b}$, because the energymomentum tensor of the matter induced in $4 D$ is given by [22]

$$
8 \pi T_{\mu \nu} \sim \frac{\Phi_{\mu ; \nu}}{\Phi}
$$

for any value of $N$. Thus, substituting (28) into (11) and (2) we obtain that the effective mass measured by an observer in $4 D$, is determined by the extra dimension.

\subsection{The gravitational mass}

Tolman-Whittaker approach: In general relativity, when the field is time-independent, the gravitational mass inside a $3 D$ volume $V_{3}$, is given by the Tolman-Whittaker formula (1). Using the Einstein field equations, $8 \pi T_{\mu \nu}=$ $G_{\mu \nu}$, it can be expressed in terms of the metric coefficients. In the present case the exterior metric has the form

$$
d s^{2}=e^{\nu(r)} d t^{2}-e^{\lambda(r)} d r^{2}-r^{2} e^{\mu(r)}\left[d \theta^{2}+\sin ^{2} \theta d \phi^{2}\right]
$$

for which we find

$$
M_{g}(r)=\frac{1}{2} r^{2} e^{(\mu-\lambda / 2+\nu / 2)} \nu^{\prime},
$$

where the prime ' denotes derivative with respect to $r$. We note that this is equivalent to substituting (28) into (1) 22]. Evaluating this expression for the metric (17) we obtain

$$
M_{g}(r)=\varepsilon \mathcal{M}\left(1-\frac{2 \mathcal{M}}{r}\right)^{b(N-1) / 2}
$$

Here, we require $\varepsilon \mathcal{M}>0$ in order to ensure the positivity of $M$. Therefore, the total gravitational mass, measured by an observer located at infinity, is given by

$$
M_{g}=\varepsilon \mathcal{M}
$$


Low velocity approximation of the geodesic equation: This result can also be obtained from the analysis of the geodesic motion. In fact, let us consider the radial motion of a test particle in the gravitational field described by the effective metric (17). For the line element (29), the locally measured radial velocity $V$ and the locally measured radial acceleration $g$ are given by [30]

$$
V=\frac{e^{\lambda / 2} d r}{e^{\nu / 2} d t}, \quad g=\frac{d V}{e^{\nu / 2} d t}
$$

From the zeroth component of the geodesic equation we get

$$
\frac{d t}{d s}=C e^{-\nu}
$$

where $C$ is a constant of integration. From (29) evaluated at $\theta=\phi=$ constant we obtain

$$
C^{2}=\frac{e^{\nu}}{1-V^{2}}
$$

Thus, $V=\sqrt{1-e^{\nu} / C^{2}}$. Taking the time derivative of this and using (35), we find

$$
g=-\left(1-V^{2}\right) e^{-\lambda / 2} \frac{\nu^{\prime}}{2}
$$

which for the effective metric (17) yield:5

$$
g=-\left(1-V^{2}\right) \frac{\varepsilon \mathcal{M}}{R^{2}} A^{[-\varepsilon+b(N-1)] / 2} .
$$

For $\varepsilon=1$ it reproduces the usual Schwarzschild expression. For a test body, far from the center, either at rest at a point $R$ or in the low velocity approximation $(|V| \ll 1)$, (37) reduces to

$$
g=-\frac{\varepsilon \mathcal{M}}{R^{2}}
$$

which is the usual Newtonian acceleration of gravity produced by a central body of gravitational mass $\varepsilon \mathcal{M}$, in agreement with (32).

\subsection{The inertial mass: weak field limit}

An excellent analysis of the formulae defining the energy, momentum, and angular momentum for gravitational systems can be found in Weinberg's book Gravitation and Cosmology [31. In appendix B, we follow that analysis and adapt it to our 6 notation and conventions for the signature of the metric and definition of various quantities in the theory.

The inertial mass is the mass that appears in the four-momentum vector of the body. In particular, for an isolated body

$$
P^{\mu}=\left(M_{i n}, 0,0,0\right) .
$$

So what we want to calculate is $P^{0}$. In order to simplify the notation, let us write the line element of the effective spacetime (17) as

$$
d s_{(N)}^{2}=A^{\varepsilon} d t^{2}-A^{p} d r^{2}-r^{2} A^{q}\left[d \theta^{2}+\sin ^{2} \theta d \phi^{2}\right]
$$

with

$$
p=-\varepsilon+b(N-1), \quad q=1-\varepsilon+b(N-1), \quad q-p=1 .
$$

\footnotetext{
${ }^{5}$ Using (30) it takes a more familiar form. Namely, $g=-\left(1-V^{2}\right) e^{-\nu / 2} M_{g} / R^{2}$.

${ }^{6}$ For consistency, in our research we always use the conventions and definitions found in Landau and Lifshitz [1]. It should be noted that in the cited edition a sign has been changed in the definition of the electromagnetic field stress tensor.
} 
First we change coordinates from $(r, \theta, \phi)$ to $(x, y, z)$

$$
x=r \sin \theta \cos \phi, \quad y=r \sin \theta \sin \phi, \quad z=r \cos \theta, \quad r=\sqrt{x^{2}+y^{2}+z^{2}},
$$

and denote $x=x^{1}, y=x^{2}, z=x^{3}$. Using the Minkowski metric

$$
\eta_{\mu \nu}=\operatorname{diag}(1,-1,-1,-1),
$$

we have $x_{i}=-x^{i}$ and

$$
-\eta_{i j} d x^{i} d x^{j}=d x^{2}+d y^{2}+d z^{2}=d r^{2}+r^{2}\left[d \theta^{2}+\sin ^{2} \theta d \phi^{2}\right] .
$$

In these new coordinates, and notation, the spatial part of the metric (40) becomes

$$
g_{i j}=-A^{p}(1-A) n_{i} n_{j}+A^{q} \eta_{i j},
$$

where

$$
n^{i}=\frac{x^{i}}{r}, \quad n_{i}=\frac{x_{i}}{r}=-\frac{\partial r}{\partial x^{i}}, \quad n_{i} n^{i}=-1 .
$$

In order to calculate the total mass (B-22), we need the asymptotic behavior of the spatial part of $h_{\mu \nu}$ defined in (B-1). Thus, as $r \rightarrow \infty$

$$
h_{i j}=g_{i j}-\eta_{i j} \rightarrow-\frac{2 \mathcal{M}}{r}\left(n_{i} n_{j}+q \eta_{i j}\right)+O\left(\frac{1}{r^{2}}\right) .
$$

Also,

$$
h_{i}^{i}=\eta^{j k} h_{j k} \rightarrow \frac{2 \mathcal{M}(1-3 q)}{r}+O\left(\frac{1}{r^{2}}\right)
$$

Finally, using

$$
\frac{\partial n^{i}}{\partial x^{k}}=\frac{\delta_{k}^{i}+n^{i} n_{k}}{r}, \quad \frac{\partial n_{i}}{\partial x^{k}}=\frac{\eta_{i k}+n_{i} n_{k}}{r}
$$

we obtain

$$
\frac{\partial h_{k}^{j}}{\partial x^{j}}-\frac{\partial h_{j}^{j}}{\partial x^{k}} \rightarrow-\frac{4 \mathcal{M}}{r^{2}}(1-q) n_{k}+O\left(\frac{1}{r^{3}}\right) .
$$

Substituting this into (B-22) we get the total inertial mass, viz.,

$$
M_{\text {in }}=\mathcal{M}(1-q) .
$$

Now going back to the original parameters (41) we have

$$
M_{i n}=\mathcal{M}[\varepsilon-b(N-1)] .
$$

Thus, the inertial and gravitational masses are identical if

$$
b(N-1)=0,
$$

which is the same requirement (27) needed for the compatibility of the theory with the Newtonian limit.

\subsection{The total amount of matter}

Let us now evaluate the integral (2) over the whole three-dimensional volume. In the present case, since the massenergy density outside of a star does not vanish, it separates into two parts,

$$
m=4 \pi \int_{0}^{\infty} R^{2} \rho d R=4 \pi \int_{0}^{R_{b}} \bar{R}^{2} \rho_{\text {in }}(\bar{R}) d \bar{R}+4 \pi \int_{r_{b}}^{\infty} R^{2} \rho_{\text {out }}(r) d R .
$$


In the r.h.s., the first integral represents the amount of matter 7 inside of the star. Using that

$$
8 \pi \rho_{i n}=-e^{-\sigma}\left(\frac{1}{R^{2}}-\frac{\sigma_{R}}{R}\right)+\frac{1}{R^{2}}
$$

where $\sigma_{R}=d \sigma / d R$, and "in" indicates the matter quantities inside of the star, it can be written as

$$
m_{i n}=4 \pi \int_{0}^{R_{b}} \bar{R}^{2} \rho_{i n}(\bar{R}) d \bar{R}=\frac{1}{2}\left[\bar{R}\left(1-e^{-\sigma(\bar{R})}\right)\right]_{0}^{R_{b}}=\frac{R_{b}}{2}\left(1-e^{-\sigma\left(R_{b}\right)}\right) .
$$

The second integral in the r.h.s. of (53) represents the amount of matter outside of the star. In order to calculate it we use $R=r e^{\mu / 2}$ and

$$
8 \pi \rho_{\text {out }}=-e^{-\lambda}\left(\mu^{\prime \prime}+\frac{3}{4} \mu^{\prime 2}+\frac{3 \mu^{\prime}}{r}+\frac{1}{r^{2}}-\frac{\lambda^{\prime} \mu^{\prime}}{2}-\frac{\lambda^{\prime}}{r}\right)+\frac{e^{-\mu}}{r^{2}} .
$$

We find

$$
m_{\text {out }}=4 \pi \int_{r_{b}}^{\infty} R^{2} \rho_{\text {out }}(r) d R=\frac{1}{2}\left[r e^{\mu / 2}\left(1-e^{\mu-\lambda}\left(1+\frac{r \mu^{\prime}}{2}\right)^{2}\right)\right]_{r_{b}}^{\infty} .
$$

Using the boundary condition (A-7) we obtain

$$
m_{\text {out }}=\mathcal{M}[\varepsilon-b(N-1)]-\frac{R_{b}}{2}\left[1-e^{-\sigma\left(R_{b}\right)}\right] .
$$

Thus,

$$
m=m_{\text {in }}+m_{\text {out }}=\mathcal{M}[\varepsilon-b(N-1)] .
$$

This is identical to (52). Thus, the total amount of matter is equivalent to the total inertial mass (52), for any value of parameters $\varepsilon$ and $N$.

\section{The dominant energy condition}

It should be noted that the effective matter quantities do not have to satisfy the regular energy conditions [32]. However, in this section we point out an interesting link between the dominant energy condition for the effective matter and the weak equivalence principle.

An observer in $4 D$ who is not aware of the existence of an extra dimension will interpret the metric (17) as if it were governed by an effective energy-momentum tensor, which is discussed in the appendix of Ref. [9]. It is easily seen that the stresses and the energy density decrease at different rates as we move outward. To simplify the discussion, we restate the asymptotic form of the effective energy-momentum tensor. As $r \rightarrow \infty$, they are

$$
\begin{aligned}
8 \pi T_{0}^{0} & \rightarrow \frac{\mathcal{M}^{2}\left\{\left(1-\varepsilon^{2}\right)+b(N-1)[2 \varepsilon-b(N-1)]\right\}}{r^{4}}, \\
8 \pi T_{1}^{1} & \rightarrow-\frac{2 \mathcal{M} b(N-1)}{r^{3}}-\frac{\mathcal{M}^{2}\left\{\left(1-\varepsilon^{2}\right)-b(N-1)[2-b(N-1)]\right\}}{r^{4}}, \\
8 \pi T_{2}^{2} & \rightarrow \frac{\mathcal{M} b(N-1)}{r^{3}}+\frac{\mathcal{M}^{2}\left[\left(1-\varepsilon^{2}\right)-b(N-1)\right]}{r^{4}} .
\end{aligned}
$$

Thus, for an arbitrary $N$, the stresses decrease as $1 / r^{3}$, whereas the energy density goes as $1 / r^{4}$. Therefore, at large distances from the source $\left|T_{1}^{1}\right| \gg T_{0}^{0}$ and $\left|T_{2}^{2}\right|=\left|T_{3}^{3}\right| \gg T_{0}^{0}$. However, we note that if

$$
b(N-1)=0,
$$

then, the effective matter quantities satisfy the dominant energy condition $\left(T_{0}^{0} \geq\left|T_{1}^{1}\right|\right.$ and $\left.T_{0}^{0} \geq\left|T_{2}^{2}\right|=\left|T_{3}^{3}\right|\right)$ everywhere. Thus, the equality of all masses (4) guarantees not only the existence of a Newtonian limit (27) but also the fulfillment of the dominant energy condition for the effective matter.

\footnotetext{
${ }^{7}$ In order to avoid a misunderstanding, here by "amount of matter" we mean the integral quantity (2) and not the molar mass.
} 


\section{The effective exterior metric for $N=1$ and $N=0$}

In this section we compare and contrast the effective exteriors produced by the factorizations with $N=1$ and $N=0$.

\section{$5.1 \quad N=1$}

The factorization with $N=1$ leads to a family of non-Schwarzschild exteriors, namely

$$
d s_{(N=1)}^{2}=A^{\varepsilon} d t^{2}-A^{-\varepsilon} d r^{2}-A^{(1-\varepsilon)} r^{2}\left[d \theta^{2}+\sin ^{2} \theta d \phi^{2}\right],
$$

which are compatible with (i) the dominant energy condition; (ii) Newtonian physics, in the weak-field limit; (iii) the general-relativistic Schwarzschild limit for $\varepsilon=1$; and (iv) the equivalence principle:

$$
M_{g}=M_{i n}=m=\varepsilon \mathcal{M} \text {. }
$$

The only theoretical restriction on $\varepsilon$ comes from the positivity of the energy-density outside of the star. Namely,

$$
8 \pi \rho_{\text {out }}=\frac{\mathcal{M}^{2}\left(1-\varepsilon^{2}\right)}{r^{4}}\left(1-\frac{2 \mathcal{M}}{r}\right)^{(\varepsilon-2)}, 0 \leq \varepsilon \leq 1 .
$$

However, experimental and observational evidence [20, 21] suggests that $\varepsilon$ should be very close to 1, which is its Schwarzschild value.

The effective exterior with $N=1$ presents another outstanding feature. Specifically, that for the exterior matter distribution,

$$
\left(T_{0}^{0}-T_{1}^{1}-T_{2}^{2}-T_{3}^{3}\right)=0
$$

because $T_{1}^{1}=-T_{0}^{0}$ and $T_{1}^{1}=T_{2}^{2}=T_{0}^{0}$. Thus, although the exterior (62) is not empty, it shares a common property with the Schwarzschild vacuum exterior, namely, that it does not contribute to the total mass.

In order to make contact with other works in the literature, let us express the mass in terms of the original Kaluza-Klein parameters $a$ and $b$. Using (18), (32), (52) and (59) we find

$$
M_{g}=M_{i n}=m=\mathcal{M}\left(a+\frac{b}{2}\right),
$$

and in terms of the original Gross-Perry parameters $\alpha$ and $\beta$ (with $\alpha=1 / a, \beta=b / a$ )

$$
M_{g}=M_{i n}=m=\frac{\mathcal{M}(2+\beta)}{2 \alpha} .
$$

\section{$5.2 N=0$}

This is the most popular interpretation in the literature, where the metric induced on the subspace $y=$ constant is interpreted as the effective metric in $4 D$. Namely,

$$
d s_{(N=0)}^{2}=A^{a} d t^{2}-A^{-(a+b)} d r^{2}-r^{2} A^{(1-a-b)}\left[d \theta^{2}+\sin ^{2} \theta d \phi^{2}\right] .
$$

This metric has been used in the discussion of many observational problems, which include the classical tests of relativity, as well as the geodesic precession of a gyroscope. It predicts a departure from the equivalence principle, viz.,

$$
M_{g}=a \mathcal{M}, \quad M_{i n}=m=\mathcal{M}(a+b) .
$$

Notice that the inertial mass in symmetric with respect to $a$ and $b$, which responds to the fact that the spatial part of (68) is invariant under $a \leftrightarrow b$. In terms of Gross-Perry parameters

$$
M_{g}=\frac{\mathcal{M}}{\alpha}, \quad M_{i n}=m=\frac{\mathcal{M}(1+\beta)}{\alpha} .
$$

Thus, only for $b=\beta=0$, which corresponds to the Schwarzschild vacuum exterior, are the masses equal.

The line element (68) is incompatible with the Newtonian limit. Besides, at large distances from the origin the effective matter quantities do not satisfy the dominant energy condition. 


\section{The Deser-Soldate definition of mass}

In a well-known paper [10, Deser and Soldate propose a definition for the total energy $P^{0}$, which explicitly contains a contribution from the extra dimension (equation 2.7 in that paper). In our notation, it is

$$
M_{D S}=\frac{1}{16 \pi} \int\left(\frac{\partial h_{k}^{(i n d) j}}{\partial x^{j}}-\frac{\partial h_{j}^{(i n d) j}}{\partial x^{k}}-\frac{\partial h_{5}^{5}}{\partial x^{k}}\right) r^{2} n^{k} d \Omega .
$$

In this section we show that Deser-Soldate's mass definition is equivalent to the inertial mass $(\bar{B}-22)$ for the conformal factor $N=1$. The discussion in this section is general.

\subsection{General formulae}

Let us apply (B-22) to the case where

$$
g_{\mu \nu} \equiv g_{\mu \nu}^{(e f f)}=\Phi^{N} g_{\mu \nu}^{(i n d)}, \quad \epsilon \Phi^{2}=g_{55},
$$

where $g_{55}$ is the metric coefficient in front of the extra dimension, and $\epsilon= \pm 1$, depending on whether it is spacelike or timelike 8 . Therefore, the Minkowski metric in $5 D$ is $\eta_{A B}=\operatorname{diag}(1,-1,-1,-1, \epsilon)$. Now, setting $g_{55}=h_{55}+\eta_{55}$, the asymptotic behavior, as $r \rightarrow \infty$, of the effective metric (72) is given by

$$
g_{\mu \nu} \equiv g_{\mu \nu}^{(e f f)} \rightarrow h_{\mu \nu}^{(i n d)}+\frac{N}{2} \epsilon h_{55} \eta_{\mu \nu}+\eta_{\mu \nu}
$$

where $h_{\mu \nu}^{i n d}$ represents the asymptotic form of the induced metric. In other words, in this limit

$$
h_{\mu \nu} \equiv h_{\mu \nu}^{(e f f)} \rightarrow h_{\mu \nu}^{(i n d)}+\frac{N}{2} \epsilon h_{55} \eta_{\mu \nu}
$$

Consequently,

$$
\left(\frac{\partial h_{k}^{(e f f) j}}{\partial x^{j}}-\frac{\partial h_{j}^{(e f f) j}}{\partial x^{k}}\right) \rightarrow\left(\frac{\partial h_{k}^{(i n d) j}}{\partial x^{j}}-\frac{\partial h_{j}^{(i n d) j}}{\partial x^{k}}-N \frac{\partial h_{5}^{5}}{\partial x^{k}}\right) .
$$

Thus, the total inertial mass becomes

$$
M_{i n}=\frac{1}{16 \pi} \int\left(\frac{\partial h_{k}^{(i n d) j}}{\partial x^{j}}-\frac{\partial h_{j}^{(i n d) j}}{\partial x^{k}}-N \frac{\partial h_{5}^{5}}{\partial x^{k}}\right) r^{2} n^{k} d \Omega,
$$

which depends explicitly on $h_{5}^{5}$ and $N$. First, note that this result holds regardless of the signature of the extra dimension. Secondly, for $N=1$, this equation becomes identical to (71), which is the flux integral expression proposed by Deser and Soldate [10]

The above shows that the Deser-Soldate definition of inertial mass should be interpreted as the total inertial mass of classical gravitational systems whose spacetime is conformal to the induced metric, with $\Phi=\sqrt{\epsilon g_{55}}$, i.e. $N=1$, as a conformal factor. Indeed, Deser and Soldate indicate that $h_{55}$ can be removed from (76) through a conformal rescaling of the spacetime (induced) metric, to yield a form like (B-22) without the last term. However, they do not elaborate much about this.

\footnotetext{
${ }^{8}$ Do not confuse $\varepsilon$ with $\epsilon$
} 


\subsection{Example}

Deser and Soldate applied their definition of total energy to the family of spherically symmetric exterior solutions (7) in the form given by Gross and Perry,

$$
d S^{2}=\left(\frac{1-\mathcal{M} / 2 \rho}{1+\mathcal{M} / 2 \rho}\right)^{2 / \alpha} d t^{2}-\left(\frac{1-\mathcal{M} / 2 \rho}{1+\mathcal{M} / 2 \rho}\right)^{2(\alpha-\beta-1) / \alpha}\left(1+\frac{\mathcal{M}}{2 \rho}\right)^{4}\left[d \rho^{2}+\rho^{2} d \Omega^{2}\right] \pm\left(\frac{1-\mathcal{M} / 2 \rho}{1+\mathcal{M} / 2 \rho}\right)^{2 \beta / \alpha} d y^{2},
$$

and obtained

$$
M_{D S}=\frac{\mathcal{M}(2+\beta)}{2 \alpha}
$$

which is identical to (67), instead of $M_{\text {in }}=\mathcal{M}(1+\beta) / \alpha$ corresponding to $N=0$. If we apply the general expression (76) to Gross-Perry solution we get $M_{D S}=(\mathcal{M} / \alpha)[1+\beta(1-N / 2)]$, which becomes identical to (52), after changing $\alpha=1 / a, \beta=b / a$. Thus, for $N=1$

$$
M_{g}=M_{i n}=m=M_{D S}=\varepsilon \mathcal{M} .
$$

In summary, (76) gives the inertial mass of a gravitational system whose effective spacetime is conformal to the induced metric, $g_{\mu \nu}^{(e f f)}=\Phi^{N} g_{\mu \nu}^{(i n d)}$ with $\Phi=\sqrt{\epsilon g_{55}}$. Regarding our particular example, it should be noted that frequently in the literature, (77) is interpreted as the expression for the inertial mass of the effective spacetime with $N=0$ (instead of $N=1)$. Our calculations show that such interpretation is inappropriate.

\section{Summary and concluding remarks}

The $(4+1)$ dimensional reduction of the field equations in $5 D$ leads to a set of equations in $4 D$ which is not closed: the effective equations for gravity in $4 D$ are weaker than in ordinary general relativity [7. This reflects the fact that there are many ways of producing, or embedding, a $4 D$ spacetime in a given higher-dimensional manifold, while satisfying the field equations [33. As a consequence, the effective picture in four dimensions allows the existence of different possible non-Schwarzschild scenarios for the description of the spacetime outside of a spherical star 9], 34].

In this paper we have examined the equivalence principle mainly in the sense where it refers to the equality of definitions for mass which are based on a metric, in the tradition of Deser and Soldate [10, Davisson and Owen [12] and Gross and Perry [14]. The main question under investigation has been whether the violation of this principle is a necessary consequence of the existence of extra dimensions.

In this context, we have shown that the line element

$$
d s_{(N=1)}^{2}=\left(1-\frac{2 \mathcal{M}}{r}\right)^{\varepsilon} d t^{2}-\left(1-\frac{2 \mathcal{M}}{r}\right)^{-\varepsilon} d r^{2}-\left(1-\frac{2 \mathcal{M}}{r}\right)^{(1-\varepsilon)} r^{2}\left[d \theta^{2}+\sin ^{2} \theta d \phi^{2}\right]
$$

is consistent with the weak equivalence principle (44), the Newtonian limit, and the dominant energy condition for any value of the free parameter $\varepsilon$. In fact, all definitions of mass lead to the same result, namely, $M_{g}=M_{i n}=m=$ $M_{D S}=\varepsilon \mathcal{M}$. We note that the range of values of parameter $\varepsilon$ can be restricted to $0 \leq \varepsilon \leq 1$ if one assumes that the external effective matter density (64) is positive. For $\varepsilon=1$ we recover the Schwarzschild solution. We stress the fact that the masses are equal even in the non-Schwarzschild case where $\varepsilon \neq 1$. We have not discussed here any particular $5 D$ embedding for this metric but the existence of such an embedding is guaranteed by Campbell-Maagard's embedding theorems [37], [38].

Although observations suggest that $\varepsilon$ must be very close to 1 , as a matter of principle let us note that for $\varepsilon=0$ we get a spacetime whose total inertial and gravitational mass is zero, but which is not flat in $4 D$. Indeed, the Riemann tensor, for $\varepsilon=0$, has the following nonzero components

$$
R_{1212}=\frac{R_{1313}}{\sin ^{2} \theta}=-\frac{\mathcal{M}^{2}}{r^{2} A}, \quad R_{2323}=\mathcal{M}^{2} \sin ^{2} \theta .
$$

Coming back to our problem, let us notice that the requirement that in the weak-field limit we recover the usual Newtonian physics, by virtue of (27), is equivalent to demand the equality (4) among all the masses, as well as the 
fulfillment of the dominant energy condition (61). The opposite is also true, the dominant energy condition demands the equality of masses and guarantees the validity of the Newtonian limit.

What this mean is that, at least within the context of Kaluza-Klein theory, the existence of an extra dimension does not necessarily imply a major departure from some basic principles of general relativity. In fact, the line element (79) is compatible with (i) Newtonian physics, in the weak-field limit; (ii) the general-relativistic Schwarzschild limit for $\varepsilon=1$; (iii) the dominant energy condition, and (iv) the equivalence principle.

The information about the extra dimension is consolidated in the non-local stresses induced in $4 D$ from the Weyl curvature tensor in $5 D$. Thus, the exterior of a star is not absolutely empty as in the Schwarzschild vacuum exterior, but is surrounded by a cloud of matter (64) that decreases as $1 / r^{4}$. The relevant characteristic of this cloud is that it has no effect on gravitational interactions. In fact, it satisfies $\left(T_{0}^{0}-T_{1}^{1}-T_{2}^{2}-T_{3}^{3}\right)=0$, which corresponds to $M_{g}=0$. In other words, the region outside the surface of a star, described by (79), does not contribute to the total mass, similarly to what happens in ordinary general relativity, where outside the surface $T_{0}^{0}=T_{1}^{1}=T_{2}^{2}=T_{3}^{3}=0$.

Thus, the exterior metric (79) is Schwarzschild-like in many aspects. However, this does not mean that the putative extra dimension has no effects in $4 D$.

1. The meaning of the equivalence principle in 5D theories of gravity has been discussed by Wesson [17] (pp. 85-88). He pointed out that in more than 4-dimensions, the definition of the principle becomes semantological, and that the geodesics followed (or not) by test particles may be used to rule out some versions of Kaluza-Klein gravity [36], [35]. In experiments involving the motion of test particles, in principle, one should be able to measure some anomalous acceleration. From (37), with $|V| \ll 1$ and $N=1$, we get

$$
\left.g=-\frac{\mathcal{M}}{r^{2} \sqrt{A}}\left\{1+\frac{\ln A+2}{2}(\varepsilon-1)+\frac{\ln A(\ln A+4)}{8}(\varepsilon-1)^{2}+O\left((\varepsilon-1)^{3}\right)\right)\right\},
$$

which an observer in $4 D$ could interpret as a violation of the equivalence principle. However, this is not a violation of (4) but a departure from the usual $(4 D)$ law of geodesic motion, which can be interpreted as some sort of non-gravitational force 9 (In this sense "the problem is largely one of semantics" [17]).

2. In the context of the stellar structure, the deviation from the Schwarzschild vacuum exterior affects the parameters of a neutron star. The general relativity upper limit $M / R<4 / 9$ is significantly increased as we go away from the Schwarzschild vacuum exterior. In principle, the compactness limit of a star can be larger than $1 / 2$, without being a black hole. Again, more work is needed in this area.

Also, we have clarified the meaning of the Deser-Soldate definition of total energy, which is usually interpreted as the total mass (or energy) of a spacetime whose line element is the induced metric, i.e., $g_{\mu \nu}=g_{\mu \nu}^{(i n d)}$. We have shown that it corresponds to a spacetime with metric $g_{\mu \nu}=\sqrt{\varepsilon g_{55}} g_{\mu \nu}^{(i n d)}$.

We would like to finish this paper with the following remarks. Theories in $5 D$ allow the existence of different, nonSchwarzschild, scenarios for the description of the spacetime outside of a spherical star, contrary to four-dimensional general relativity, where we have only one possibility which is the Schwarzschild exterior metric. Then, an observer in $4 D$, who is not directly aware of the existence of an extra dimension, will interpret these non-Schwarzschild exteriors as if they were governed by an effective energy-momentum tensor.

For example, the vacuum solutions discussed in references [2]-8, have non-vanishing effective energy-momentum tensors, which are (as usually) defined through the conventional Einstein equations. In [8], among other things, the authors present the explicit form of the effective matter quantities for various non-Schwarzschild vacuum exteriors. These are not zero, in particular $\rho_{\text {out }} \neq 0$, but are "quite exotic" from the viewpoint of the energy conditions. Certainly, they do not represent any kind of "regular" (or baryonic) matter that we know of; their nature is purely geometrical.

In the case of the Kaluza-Klein models under consideration we have seen in section 4 that the dominant energy condition is not an additional assumption but a consequence of the fulfillment of the equivalence principle. The effective matter outside of the star is gravitationally innocuous, in the sense that the corresponding gravitational

\footnotetext{
${ }^{9}$ Like the so-called "fifth" force
} 
mass is zero (equations (10) and (65)) as if there were no matter at all. However, as discussed above in (81) it should affect the motion of test particles as well as the stellar structure [9].

Acknowledgment: I would like to thank Paul Wesson for his comments on the meaning of the equivalence principle in more than 4-dimensions.

\section{Appendix A: Boundary conditions}

In general relativity, a covariant presentation of the matching conditions, across a separating hypersurface, requires the continuity of the first and second fundamental forms. However, for every particular line element these conditions might look quite differently. Here we present them for the metrics under consideration.

The interior of a spherical star will be described by the static line element

$$
d s^{2}=e^{\omega(R)} d t^{2}-e^{\sigma(R)} d R^{2}-R^{2}\left[d \theta^{2}+\sin \theta d \phi^{2}\right],
$$

while the spacetime outside of a star is assumed to pertain to the family given by (17), which has the form

$$
d s^{2}=e^{\nu(r)} d t^{2}-e^{\lambda(r)} d r^{2}-r^{2} e^{\mu(r)}\left[d \theta^{2}+\sin ^{2} \theta d \phi^{2}\right] .
$$

The exterior boundary of the star is a three-dimensional surface $\Sigma$ defined as $R=R_{b}$, and $r=r_{b}$ from inside and outside, respectively.

Standard matching conditions require continuity of the metric at $\Sigma$. Namely,

$$
e^{\omega\left(R_{b}\right)}=e^{\nu\left(r_{b}\right)}, \quad R_{b}=r_{b} e^{\mu\left(r_{b}\right) / 2} .
$$

If $n_{\mu}=\delta_{\mu}^{1} \sqrt{-g_{11}}$ represents the unit vector orthogonal to the boundary surface, then the second fundamental form (say $d K^{2}$ ) calculated from inside and evaluated at $\Sigma_{\mid R_{b}}$ gives

$$
d K_{\mid \Sigma_{R_{b}}}^{2}=-e^{-\sigma\left(R_{b}\right) / 2}\left\{\frac{1}{2} \omega_{R}\left(R_{b}\right) e^{\omega\left(R_{b}\right)} d t^{2}-R_{b}\left[d \theta^{2}+\sin ^{2} \theta d \phi^{2}\right]\right\} .
$$

From outside yields

$$
d K_{\mid \Sigma_{r_{b}}}^{2}=-e^{-\lambda\left(r_{b}\right) / 2}\left\{\frac{1}{2} \nu_{r}\left(r_{b}\right) e^{\nu\left(r_{b}\right)} d t^{2}-\left(\frac{1}{r_{b}}+\frac{1}{2} \mu_{r_{b}}\left(r_{b}\right)\right) r_{b}^{2} e^{\mu\left(r_{b}\right)}\left[d \theta^{2}+\sin ^{2} \theta d \phi^{2}\right]\right\}
$$

Now, the continuity of the second fundamental form requires

$$
e^{-\sigma\left(R_{b}\right) / 2} \omega_{R}\left(R_{b}\right)=e^{-\lambda\left(r_{b}\right) / 2} \nu_{r}\left(r_{b}\right),
$$

and

$$
e^{-\sigma\left(R_{b}\right) / 2}=e^{-\lambda\left(r_{b}\right) / 2}\left(\frac{1}{r_{b}}+\frac{\mu_{r}\left(r_{b}\right)}{2}\right) r_{b} e^{\mu\left(r_{b}\right) / 2} .
$$

Inside the fluid sphere the gravitational mass (within a sphere of radius $R$ ) and the radial "pressure" $p_{r}==-T_{1}^{1}$ are given by

$$
\begin{gathered}
M_{g}(R)=\frac{1}{2} R^{2} e^{(\omega-\sigma) / 2} \omega_{R}, \\
8 \pi p_{r}=e^{-\sigma}\left(\frac{\omega_{R}}{R}+\frac{1}{R^{2}}\right)-\frac{1}{R^{2}} .
\end{gathered}
$$

Meanwhile, outside of the sphere we have

$$
M_{g}(r)=\frac{1}{2} r^{2} e^{(\mu+\nu-\lambda) / 2} \nu_{r}
$$


and

$$
8 \pi p_{r}=e^{-\lambda}\left(\frac{\nu_{r}+\mu_{r}}{r}+\frac{\nu_{r} \mu_{r}}{2}+\frac{\mu_{r}^{2}}{4}+\frac{1}{r^{2}}\right)-\frac{e^{-\mu}}{r^{2}} .
$$

It is clear that the continuity of the first and second fundamental forms across the boundary surface guarantees the continuity of the gravitational mass and radial pressure.

\section{Appendix B: Definition of total energy}

When applying the formalisms of general relativity one has to take special care of conventions and definitions. Some authors work with signature $(+,-,-,-)$, and others with $(-,+,+,+)$. Also, there are different definitions for the Riemann-Christoffel curvature tensor. As a consequence the Einstein field equations look different. For example, $G_{\mu \nu}=-8 \pi G T_{\mu \nu}$ in [31] and $G_{\mu \nu}=8 \pi G T_{\mu \nu}$ in [1]. Besides, the quantity $Q^{\alpha \beta \gamma}$ (see bellow) is defined with different symmetry properties. The object of this appendix is to make sure we are using the "correct" equations. Therefore, we follow Weinberg's neat presentation [31, but use the conventions of Landau and Lifshitz [1].

Let us start by decomposing the spacetime metric into its asymptotic value $\eta_{\mu \nu}$, which is the Minkowski metric (43), and a deviation $h_{\mu \nu}$, viz.,

$$
g_{\mu \nu}=h_{\mu \nu}+\eta_{\mu \nu}
$$

The part of the Ricci tensor linear in $h_{\mu \nu}$ is

$$
R_{\mu \nu}^{(1)}=\frac{1}{2}\left(\frac{\partial^{2} h_{\mu}^{\alpha}}{\partial x^{\nu} \partial x^{\alpha}}+\frac{\partial^{2} h_{\nu}^{\alpha}}{\partial x^{\mu} \partial x^{\alpha}}-\frac{\partial^{2} h_{\alpha}^{\alpha}}{\partial x^{\mu} \partial x^{\nu}}-\eta^{\alpha \beta} \frac{\partial^{2} h_{\mu \nu}}{\partial x^{\alpha} \partial x^{\beta}}\right),
$$

where $h_{\beta}^{\alpha}=\eta^{\alpha \rho} h_{\rho \beta}$ and $h_{\alpha}^{\alpha}=\eta^{\alpha \beta} h_{\alpha \beta}$. Now,

$$
R_{\mu \nu}^{(1)}-\frac{1}{2} \eta_{\mu \nu} R^{(1)}=8 \pi\left(T_{\mu \nu}+t_{\mu \nu}\right),
$$

with $R^{(1)}=\eta^{\mu \nu} R_{\mu \nu}^{(1)}$ and

$$
t_{\mu \nu}=\frac{1}{8 \pi}\left[\left(R_{\mu \nu}^{(1)}-\frac{1}{2} \eta_{\mu \nu} R^{(1)}\right)-\left(R_{\mu \nu}-\frac{1}{2} g_{\mu \nu} R\right)\right]
$$

where we have used the field equations

$$
R_{\mu \nu}-\frac{1}{2} g_{\mu \nu} R=8 \pi T_{\mu \nu}
$$

Let us denote

$$
G_{\nu}^{(1) \mu} \equiv R_{\nu}^{(1) \mu}-\frac{1}{2} \delta_{\nu}^{\mu} R^{(1)}
$$

Thus, using (B-2)

$$
G^{(1) \mu \nu}=\frac{1}{2}\left(\eta^{\mu \alpha} \frac{\partial^{2} h^{\nu \beta}}{\partial x^{\alpha} \partial x^{\beta}}+\eta^{\nu \beta} \frac{\partial^{2} h^{\mu \alpha}}{\partial x^{\alpha} \partial x^{\beta}}+\eta^{\mu \nu} \eta^{\alpha \beta} \frac{\partial^{2} h_{\lambda}^{\lambda}}{\partial x^{\alpha} \partial x^{\beta}}-\eta^{\mu \alpha} \eta^{\nu \beta} \frac{\partial^{2} h_{\lambda}^{\lambda}}{\partial x^{\alpha} \partial x^{\beta}}-\eta^{\alpha \beta} \frac{\partial^{2} h^{\mu \nu}}{\partial x^{\alpha} \partial x^{\beta}}-\eta^{\mu \nu} \frac{\partial^{2} h^{\alpha \beta}}{\partial x^{\alpha} \partial x^{\beta}}\right) .
$$

Notice that

$$
\frac{\partial}{\partial x^{\nu}} G^{(1) \mu \nu}=\frac{\partial}{\partial x^{\mu}} G^{(1) \mu \nu}=0 .
$$

Therefore, the r.h.s. of (B-3)

$$
\mathcal{T}^{\mu \nu}=\eta^{\mu \lambda} \eta^{\nu \rho}\left[T_{\lambda \rho}+t_{\lambda \rho}\right]
$$

is a locally conserved quantity, i.e.,

$$
\frac{\partial}{\partial x^{\mu}} \mathcal{T}^{\mu \nu}=\frac{\partial}{\partial x^{\nu}} \mathcal{T}^{\mu \nu}=0 .
$$


There are a number of reasons 31] which suggest that $\mathcal{T}^{\mu \nu}$ should be interpreted as the total energy-momentum pseudo-tensor of matter and gravitation, and $t_{\mu \nu}$ as the energy-momentum pseudo-tensor of the gravitational field itself [1].

In addition the derivatives $\left(\partial / \partial x^{\alpha}\right)$ and $\left(\partial / \partial x^{\beta}\right)$ appear in all terms of (B-7). Then, by virtue of the constancy of the metric coefficients $\eta_{\mu \nu}$, one can factorize one, or both, of them10. The theoretical working looks a little different, but the final result is the same.

Factorizing $\partial / \partial x^{\alpha}:$ If we choose to factorize $\left(\partial / \partial x^{\alpha}\right)$, then we obtain

$$
G^{(1) \mu \nu}=8 \pi \mathcal{T}^{\mu \nu}=\frac{\partial}{\partial x^{\alpha}} Q_{L T}^{\mu \nu \alpha},
$$

with

$$
Q_{L T}^{\mu \nu \alpha}=\frac{1}{2}\left(\eta^{\mu \alpha} \frac{\partial h^{\nu \beta}}{\partial x^{\beta}}+\eta^{\nu \beta} \frac{\partial h^{\mu \alpha}}{\partial x^{\beta}}+\eta^{\mu \nu} \eta^{\alpha \beta} \frac{\partial h_{\lambda}^{\lambda}}{\partial x^{\beta}}-\eta^{\mu \alpha} \eta^{\nu \beta} \frac{\partial h_{\lambda}^{\lambda}}{\partial x^{\beta}}-\eta^{\alpha \beta} \frac{\partial h^{\mu \nu}}{\partial x^{\beta}}-\eta^{\mu \nu} \frac{\partial h^{\alpha \beta}}{\partial x^{\beta}}\right) .
$$

Here $L T$ means that this quantity is antisymmetric in its last $(L)$ two $(T)$ indices. Namely,

$$
Q_{L T}^{\mu \nu \alpha}=-Q_{L T}^{\mu \alpha \nu}
$$

Thus, $\partial G^{(1) \mu \nu} / \partial x^{\nu}=0=\partial \mathcal{T}^{\mu \nu} / \partial x^{\nu}$ is satisfied automatically.

Factorizing $\partial / \partial x^{\beta}$ : If we choose to factorize $\left(\partial / \partial x^{\beta}\right)$, then we obtain

$$
G^{(1) \mu \nu}=8 \pi \mathcal{T}^{\mu \nu}=\frac{\partial}{\partial x^{\beta}} Q_{F T}^{\beta \mu \nu},
$$

with

$$
Q_{F T}^{\beta \mu \nu}=\frac{1}{2}\left(\eta^{\mu \alpha} \frac{\partial h^{\nu \beta}}{\partial x^{\alpha}}+\eta^{\nu \beta} \frac{\partial h^{\mu \alpha}}{\partial x^{\alpha}}+\eta^{\mu \nu} \eta^{\alpha \beta} \frac{\partial h_{\lambda}^{\lambda}}{\partial x^{\alpha}}-\eta^{\mu \alpha} \eta^{\nu \beta} \frac{\partial h_{\lambda}^{\lambda}}{\partial x^{\alpha}}-\eta^{\alpha \beta} \frac{\partial h^{\mu \nu}}{\partial x^{\alpha}}-\eta^{\mu \nu} \frac{\partial h^{\alpha \beta}}{\partial x^{\alpha}}\right) .
$$

This quantity is antisymmetric in its first (F) two (T)indices,

$$
Q_{F T}^{\beta \mu \nu}=-Q_{F T}^{\mu \beta \nu}
$$

Thus, $\partial G^{(1) \mu \nu} / \partial x^{\mu}=0=\partial \mathcal{T}^{\mu \nu} / \partial x^{\mu}$ is satisfied automatically.

From (B-10) it follows that quantity

$$
P^{\mu}=\int_{V} \mathcal{T}^{\mu 0} d x^{3}
$$

may be interpreted as the total energy-momentum pseudo-vector of the system including matter, all non-gravitational fields, and gravitation. Using (B-11) or (B-14) this becomes

$$
P^{\lambda}=\frac{1}{8 \pi} \int_{V} \frac{\partial}{\partial x^{\beta}} Q_{F T}^{\beta 0 \lambda} d x^{3}=\frac{1}{8 \pi} \int_{V} \frac{\partial}{\partial x^{\alpha}} Q_{L T}^{\lambda 0 \alpha} d x^{3} .
$$

Using Gauss's theorem, integrating over a large sphere of radius $r$ we get

$$
P^{\lambda}=-\frac{1}{8 \pi} \int Q_{F T}^{j 0 \lambda} r^{2} n_{j} d \Omega=-\frac{1}{8 \pi} \int Q_{L T}^{\lambda 0_{j}} r^{2} n_{j} d \Omega
$$

where $d \Omega=\sin \theta d \theta d \phi$ and $n^{k}$ is the unit vector pointing outward

$$
n^{k}=\frac{x^{k}}{r}, \quad \eta_{j k} n^{i} n^{k}=-1 .
$$

\footnotetext{
${ }^{10} \mathrm{As}$ is done in Landau and Lifshitz [1, where they introduce the quantity $\lambda^{\mu \nu \alpha \beta}$.
} 
The total inertial mass $M_{i n}$ is given by

$$
M_{i n}=P^{0}=-\frac{1}{8 \pi} \int Q_{F T}^{j 00} r^{2} n_{j} d \Omega=-\frac{1}{8 \pi} \int Q_{L T}^{00 j} r^{2} n_{j} d \Omega
$$

After a simple calculation we obtain

$$
M_{i n}=\frac{1}{16 \pi} \int\left(\frac{\partial h_{k}^{j}}{\partial x^{j}}-\frac{\partial h_{j}^{j}}{\partial x^{k}}\right) r^{2} n^{k} d \Omega .
$$

\section{References}

[1] L.D. Landau and E.M. Lifshitz, The Classical Theory of Fields, Fourth Edition (Butterworth-Heinemann, 2002), Eqs. (105.23) and (100.23).

[2] C. Germani and R. Maartens, Phys. Rev. D64, 124010(2001); hep-th/0107011

[3] M. Bruni, C. Germani and R. Maartens, Phys. Rev. Lett. 87, 231302(2001); gr-qc/0108013.

[4] G. Kofinas and E. Papantonopoulos, J. Cosmol. Astropart. Phys. 12, 11(2004); gr-qc/0401047.

[5] N. Dadhich, R. Maartens, P. Papadopoulos and V. Rezania, Phys.Lett. B487, 1(2000); hep-th/0003061v3.

[6] R. Casadio, A. Fabbri and L. Mazzacurati, Phys.Rev. D65, 084040(2002); gr-qc/0111072v2.

[7] M. Visser and D. L. Wiltshire, Phys.Rev. D67, 104004(2003); hep-th/0212333v2.

[8] K.A. Bronnikov, H. Dehnen and V.N. Melnikov, Phys.Rev. D68, 024025(2003); gr-qc/0304068v1.

[9] J. Ponce de Leon, Class.Quantum Grav. 24, 1755(2007); gr-qc/0701129

[10] S. Deser and M. Soldate, Nuclear Phys. B311, 739(1989).

[11] K. Lake, Class. Quantum Gravit 23, 5876(2006), gr-qc/0606005.

[12] A. Davidson and D. Owen, Phys. Lett. B 155, 247(1985).

[13] D. Kramer, Acta Phys. Polon. B2, 807(1970).

[14] D.J. Gross and M.J. Perry, Nucl. Phys. B226, 29(1983). This paper is usually cited in the context of the GPS Kaluza-Klein monopole, where G, P and S stand for Gross-Perry-Sorkin (Rafael D. Sorkin, Phys. Rev. Lett. 51, 87(1983)). However, the Kaluza-Klein monopole does not belong to the class of spacetimes investigated here.

[15] G.C. Segré, "Physics in more than four-dimensions, another look at the Kaluza-Klein theory", in Cosmology and Elementary particles. Proceedings of the first winter school of physics. World Scientific Publishing Co. Pte. Ltd. 1989.

[16] P.S. Wesson and J. Ponce de Leon, Class. Quantum Grav. 11, 1341(1994).

[17] P.S. Wesson, Space-Time-Matter (World Scientific Publishing Co. Pte. Ltd. 1999).

[18] A.G.Agnese, A.P. Billyard, H. Liu and P.S. Wesson, Gen. Rel. Gravit31,527(1999).

[19] W.N. Sajko and P.S. Wesson, Gen. Rel. Gravit. 32, 1381(2000).

[20] J.M. Overduin, Phys.Rev. D62, 102001(2000), gr-qc/0007047.

[21] Hongya Liu and J.M. Overduin, Astrophys. J. bf 538, 386(2000), gr-qc//0003034. 
[22] J. Ponce de Leon, Kaluza-Klein solitions reexamined, gr-qc/0611082. To appear in Int. J. Mod. Phys. D

[23] S.S. Kokarev, Grav. and Cosmology 1, 247(1995).

[24] S.S. Kokarev, Izvestia Vuzov (Physika) 2, 50(1996). In Russian.

[25] W.N. Sajko, Phys. Rev.D60, 104038.

[26] J. Ponce de Leon, Int.J.Mod.Phys. D11, 1355(2002); gr-qc/0105120.

[27] B. Mashhoon, H. Liu and P.S. Wesson, Phys. Lett. B 331, 305(1994).

[28] L. Dolan and M.J. Duff, Phys. Rev. Lett 52, 14(1984).

[29] J. Ponce de Leon, Mod.Phys.Lett. A16, 2291(2001); gr-qc/0111011.

[30] H. Bondi, Proc. R. Soc. London A281, 39(1964).

[31] Steven Weinberg, Gravitation and Cosmology (John Wiley and Sons, Inc. 1972).

[32] K.A. Bronnikov and S-W Kim, Phys. Rev. D67, 064027(2003); gr-qc/0212112.

[33] , J. Ponce de Leon, Class.Quant.Grav. 23, 3043(2006); gr-qc/0512067.

[34] J. Ponce de Leon, Gravitation and Cosmology 14, 65(2008); arXiv:0711.0998 [gr-qc].

[35] P. Xu and Y. Ma, Phys. Lett. B656, 165(2007), arXiv:0710.3677 [gr-qc].

[36] Y.M. Cho and D.M. Park, Gen. Rel. Grav. 23, 741(1991).

[37] S.S. Seahra and P.S. Wesson, Class.Quant.Grav. 20 1321(2003); gr-qc/0302015

[38] P.S. Wesson, "In Defense of Campbell's Theorem as a Frame for New Physics"; gr-qc/0507107 